\title{
DEC1 is positively associated with the malignant phenotype of invasive breast cancers and negatively correlated with the expression of claudin-1
}

\author{
YANG LIU $^{1,2}$, YUAN MIAO ${ }^{1,2}$, JIAN WANG $^{1,2}$, XUYONG LIN $^{1,2}$, \\ LIANG WANG $^{1,2}$, HONG-TAO XU ${ }^{1,2}$ and EN-HUA WANG ${ }^{1,2}$ \\ ${ }^{1}$ Department of Pathology, The First Affiliated Hospital and College of Basic Medical Sciences, \\ ${ }^{2}$ Institute of Pathology and Pathophysiology, China Medical University, Shenyang 110001, P.R. China
}

Received November 29, 2012; Accepted February 4, 2013

DOI: 10.3892/ijmm.2013.1279

\begin{abstract}
Differentiated embryo-chondrocyte expressed gene 1 (DEC1) is a basic helix-loop-helix transcriptional regulator, reportedly involved in cell growth, differentiation, apoptosis and tumorigenesis. In breast cancer, DEC1 expression correlates with increased malignant potential and invasiveness. Nevertheless, the detailed mechanisms by which DEC1 modulates breast cancer progression are still unclear. Claudin-1, an important tight junction protein, functions as a tumor invasion suppressor. In the present study, the relationship between DEC1 and claudin-1 in 147 cases of invasive breast ductal carcinomas was examined by immunohistochemistry. Based on the data, DEC1 expression was elevated in invasive breast ductal carcinomas and DEC1 levels were positively correlated with tumor grade $(\mathrm{P}=0.023)$. Moreover, DEC1 expression was negatively correlated with the claudin-1 level (correlation coefficient $=-0.245, \mathrm{P}=0.003$ ). We further identified that, in MCF-7 and MDA-MB-231 breast cancer cell lines, DEC1 knockdown led to the enhanced expression of claudin-1 at both the mRNA and protein levels, and reduced cell invasive capacity. Collectively, our data suggest that overexpression of DEC1 may promote the invasiveness of breast cancer through downregulation of claudin-1.
\end{abstract}

\section{Introduction}

Differentiated embryo-chondrocyte expressed gene 1 (DEC1, also known as BHLHE40/Stra13/Sharp2) is a basic helix-loophelix (bHLH) transcription factor ubiquitously expressed in both embryonic and adult tissues $(1,2)$. DEC1 exerts multiple biological functions, such as neurogenesis, apoptosis, cell

Correspondence to: Professor En-Hua Wang, Department of Pathology, The First Affiliated Hospital and College of Basic Medical Sciences, China Medical University, Shenyang 110001, P.R. China E-mail:wangeh775@gmail.com

Key words: differentiated embryo chondrocyte expressed gene 1, claudin-1, breast cancer, invasion, immunohistochemistry proliferation, cell differentiation and circadian rhythms (3-8). Recent studies have revealed that the expression of DEC1 is correlated with the malignancy of a number of cancer types (9). The expression patterns of DEC1 and their impact on tumor development are largely tumor-type specific $(1,9)$. Low DEC1 expression was found to be associated with poor histological differentiation and malignancy progression in hepatocellular carcinoma (10), whereas, overexpression of DEC1 was significantly correlated with poor survival of patients with esophageal squamous cell carcinoma following surgery (11). In breast cancer, the expression of DEC1 was found to be increased upon progression from normal tissues to invasive carcinomas (12). However, the mechanisms by which DEC1 modulates cancer progression are largely unclear.

Claudin-1 is a key component of the tight junction complex and is thereby important for maintaining tight junction barrier integrity. Downregulation of claudin-1 is intimately associated with tumorigenesis in breast $(13,14)$, prostate $(15)$ and melanocytic neoplasia (16). It is postulated that the impact of claudin-1 on cancer invasion and metastasis is through its regulation of certain invasion/metastasis suppressors or enhancers $(17,18)$. As significant loss of claudin-1 expression and overexpression of DEC1 are commonly observed in the progression of various types of cancers including breast cancer, we aimed to ascertain whether DEC1 modulates breast cancer invasion through its regulation of claudin-1. Our study revealed that DEC1 modulates breast cancer invasion through regulation of claudin-1.

\section{Materials and methods}

Patients and tissue samples. A total of 147 invasive ductal breast carcinoma tissue samples were obtained from patients who underwent surgery at the First Affiliated Hospital of China Medical University, China, between 2005 and 2009. Informed consent was obtained prior to surgery from all enrolled patients. Formalin-fixed paraffin-embedded sections of tissues obtained from surgical samples were stained routinely with hematoxylin and eosin (H\&E staining), and reviewed by two senior pathologists in order to determine the histological type according to World Health Organization breast carcinoma histological classification criteria (2003). 
Accordingly, the grade of ductal carcinomas was classified into three groups: grade 1 (low grade), grade 2 (moderate grade) and grade 3 (high grade). Patients included in the study ranged from 31 to 79 years of age (mean, 51 years). All patients with tumors had axillary node status confirmed histologically. Details of the clinical data regarding age, nodal status, tumor size, grade, estrogen and progesterone receptor status of these patients are listed in Table I. The study was conducted according to the regulations stipulated by the Institution Review Board of the China Medical University.

Immunohistochemistry. All resected specimens were fixed with $10 \%$ neutral buffered formalin and embedded in paraffin blocks. Tissue blocks were sliced into $4-\mu \mathrm{m}$ sections. The sections were then deparaffinized, rehydrated and immunostained with polyclonal rabbit anti-DEC1 antibody (1:200; Novus Biologicals, Littleton, CO, USA) and polyclonal rabbit anti-claudin-1 antibody (1:100; Zymed Laboratories, Inc., South San Francisco, CA, USA) at $4^{\circ} \mathrm{C}$ overnight. Antibodies were detected by the streptavidin-peroxidase method.

Evaluation of immunostaining. All the immunostained sections were evaluated by two senior pathologists who were blinded to the clinical data. According to the previous evaluation methods, only nuclear expression of DEC1 indicates positivity (19) and $\geq 10 \%$ cytoplasm and/or membrane expression of claudin-1 indicates positivity (20).

Cell culture and treatment. The human breast cancer cell lines MCF-7 and MDA-MB-231 were obtained from the American Type Culture Collection (ATCC, Manassas, VA, USA). The cells were cultured in Dulbecco's modified Eagle's medium (DMEM)-high glucose (Sigma Chemical Co., St. Louis, MO, USA) supplemented with $10 \%$ fetal bovine serum at $37^{\circ} \mathrm{C}$ in a humidified atmosphere of $95 \%$ air and $5 \% \mathrm{CO}_{2}$.

Reverse transcription-polymerase chain reaction (RT-PCR). Total RNA was isolated using an RNeasy RNA isolation kit (Qiagen, Hilden, Germany). First-strand cDNA was synthesized from $1 \mu \mathrm{g}$ of total RNA using ReverTra Ace (Toyobo, Osaka, Japan). PCR was performed using an aliquot of first-strand cDNA as a template under standard conditions with TaqDNA polymerase (Takara, Shiga, Japan). The cDNAs for human DEC1, claudin-1 and glyceraldehyde 3-phosphate dehydrogenase (GAPDH) were amplified for up to 28, 25 and 22 cycles respectively. The primers used are listed as follows: DEC1 forward, 5'-GTGAGTCACTCTCCAGTTT-3' and reverse, 5'-ATCCGTGTCTAGCTGTGCAAT-3'; claudin-1 forward, 5'-CAGCTGTTGGGCTTCATTCTC-3' and reverse, 5'-ATC ACTCCCAGGAGGATGCC-3'; GAPDH forward, 5'-CCA CCCATGGCAAATTCCATGGCA-3' and reverse, 5'-AGA CCACCTGGTGCTCAGTGTAGC-3'. The predicted sizes of the amplified products for DEC1, claudin-1 and GAPDH were 534, 277 and $696 \mathrm{bp}$, respectively. The PCR products were separated on $1.5 \%(\mathrm{w} / \mathrm{v})$ agarose gels.

Small interfering RNA (siRNA). siRNA against DEC1 and the negative control siRNA (scrambled siRNA) were purchased from Santa Cruz Biotechnology, Inc. (catalog no. of DEC1 siRNA (h): sc-106769; catalog no. of control
siRNA-A: sc-37007). For the siRNA transfection, MCF-7 and MDA-MB-231 cells were seeded at $5 \times 10^{4}$ cells $/ 35-\mathrm{mm}$ well. Then $24 \mathrm{~h}$ later, the siRNA was transfected into the cells using Lipofectamine $^{\mathrm{TM}}$ RNAiMAX reagent (Invitrogen, Carlsbad, CA, USA). After transfection, the cells were incubated for $48 \mathrm{~h}$ and subjected to various analyses.

Western blotting. The cells transfected with siRNA or plasmid DNA were lysed using M-PER lysis buffer (Pierce, Rockford, IL, USA). Protein concentrations were determined by a bicinchoninic acid assay. The obtained lysates (20 $\mu \mathrm{g}$ protein) were subjected to SDS-PAGE, and the acquired proteins were transferred to PVDF membranes (Millipore, Billerica, MA, USA). The membranes were incubated with antibodies specific for DEC1 $(1: 20,000)$, claudin-1 $(1: 1,000)$ and actin $(1: 30,000$; Sigma Chemical Co.), followed by horseradish peroxidaseconjugated secondary antibody (Immuno-Biological Laboratories Co., Ltd., Gunma, Japan). The ECL, ECL-Plus or ECL-Advance Western Blotting Detection System (Amersham, Uppsala, Sweden) was used for detection.

Matrigel invasion assay. The Matrigen invasion assay was performed according to the manufacturer's instructions. In each upper chamber, $5 \times 10^{5}$ cells were grown in serum-free medium on $8-\mu \mathrm{m}$ porous polycarbonate membranes (Corning, Acton, MA, USA), which were coated with Matrigel basement membrane matrix (BD Biosciences, San Jose, CA, USA). The lower chambers were filled with DMEM-high glucose supplemented with $10 \%$ fetal bovine serum. After incubation for $24 \mathrm{~h}$ at $37^{\circ} \mathrm{C}$ in a humid atmosphere with $5 \% \mathrm{CO}_{2}$, the cells that had migrated through the pores were fixed with methanol for $30 \mathrm{~min}$ and stained with hematoxylin. For each filter, the numbers of cells in five different fields under x200 magnification were visualized and counted using a Nikon E200 microscope. Each experiment was performed in triplicate.

Statistical analysis. The statistical package SPSS version 13.0 for Windows (SPSS, Chicago, IL, USA) was used for all data analysis. The Chi-square test was used to assay whether the expression levels of DEC1 or claudin-1 were related to the clinicopathological characteristics of the breast cancers. The Spearman correlation test was used to examine the correlations between DEC1 and claudin-1 expression. A P-value $<0.05$ was considered to indicate a statistically significant result.

\section{Results}

Relationship between DEC1, claudin-1 expression and clinicopathological factors. We examined the expression patterns of DEC1 and claudin-1 using immunohistochemistry in 147 invasive breast ductal carcinomas. In normal human breast tissues, the expression of DEC1 was weak and patchy, and was mostly presented in the nucleus (Fig. 1A). However, in the invasive ductal breast carcinomas, the expression of DEC1 was detected in both the nucleus and the cytoplasm, and the nuclear staining was much stronger (Fig. 1B-D). The percentage of tumors positive for DEC1 was elevated as tumor grade increased; $43.75 \%$ (7/16) for grade 1, 75.96\% (75/104) for grade 2 , and $81.48 \%(22 / 27)$ for grade 3 , respectively. In 
Table I. The expression of DEC1 and claudin-1 in invasive breast ductal carcinomas.

\begin{tabular}{|c|c|c|c|c|c|c|}
\hline & \multicolumn{3}{|c|}{ DEC1 expression } & \multicolumn{3}{|c|}{ Claudin-1 expression } \\
\hline & Negative & Positive & P-value & Negative & Positive & P-value \\
\hline No. of patients & 39 & 108 & & 106 & 41 & \\
\hline Age (years) & & & 0.873 & & & 0.847 \\
\hline$<50$ & 20 & 57 & & 55 & 22 & \\
\hline$\geq 50$ & 19 & 51 & & 51 & 19 & \\
\hline Nodal status & & & 0.825 & & & 0.028 \\
\hline Negative & 31 & 84 & & 78 & 37 & \\
\hline Positive & 8 & 24 & & 28 & 4 & \\
\hline Tumor size $(\mathrm{cm})$ & & & 0.451 & & & 0.934 \\
\hline$\leq 2$ & 16 & 37 & & 38 & 15 & \\
\hline$>2$ & 23 & 71 & & 68 & 26 & \\
\hline Grade & & & 0.023 & & & 0.069 \\
\hline I & 9 & 7 & & 15 & 1 & \\
\hline II & 25 & 79 & & 72 & 32 & \\
\hline III & 5 & 22 & & 19 & 8 & \\
\hline ER status & & & 0.660 & & & 0.042 \\
\hline Negative & 16 & 40 & & 35 & 21 & \\
\hline Positive & 23 & 68 & & 71 & 20 & \\
\hline PR status & & & 0.428 & & & 0.298 \\
\hline Negative & 15 & 34 & & 38 & 11 & \\
\hline Positive & 24 & 74 & & 68 & 30 & \\
\hline
\end{tabular}

ER, estrogen receptor; $\mathrm{PR}$, progesterone receptor. $\mathrm{P}$-values in bold print indicate statistical significance at $\mathrm{P}<0.05$.

Table II. Correlations between the expression of DEC1 and claudin-1.

\begin{tabular}{lcccc}
\hline & \multicolumn{2}{c}{ Nuclear expression of DEC1 } & & \\
\cline { 3 - 4 } Cytoplasmic and/or membrane expression of claudin-1 & Negative & Positive & P-value & Correlation coefficient \\
\hline Negative & 21 & 85 & $\mathbf{0 . 0 0 3}$ & $\mathbf{- 0 . 2 4 5}$ \\
Positive & 18 & 23 & & \\
\hline
\end{tabular}

Bold values indicate statistical significance at $\mathrm{P}<0.05$.

contrast, in the normal breast tissues, claudin-1 was expressed mainly in the cytoplasm and/or on the cell membrane (Fig. 1E). Loss or attenuation of claudin-1 expression was noted in the invasive ductal breast carcinomas (Fig. 1F-H). By Chi-square analysis, we found that DEC 1 expression was correlated with tumor grade $(\mathrm{P}=0.023)$. However, no significant associations were observed in regards to patient age $(\mathrm{P}=0.873)$, lymph node status $(\mathrm{P}=0.825)$, tumor size $(\mathrm{P}=0.451)$, estrogen receptor $(\mathrm{ER})(\mathrm{P}=0.660)$ or progesterone receptor $(\mathrm{PR})(\mathrm{P}=0.428)$. Loss of claudin-1 expression was correlated with lymph node status $(\mathrm{P}=0.028)$ and $\mathrm{ER}$ status $(\mathrm{P}=0.042)$, but not with patient age $(\mathrm{P}=0.847)$, tumor size $(\mathrm{P}=0.934)$ or $\mathrm{PR}$ status $(\mathrm{P}=0.298)$. Importantly, our correlation analysis provided the evidence that there is a strong inverse correlation between DEC1 and claudin-1 expression in breast cancers $(\mathrm{P}=0.003$, correlation coefficient $=-0.245)$ (Table II).

DEC1 knockdown upregulates the expression of claudin-1 in breast cancer cell lines. MCF-7 and MDA-MB-231 breast cancer cell lines were employed to further illustrate the impact of DEC1 on claudin-1 expression. As shown in Fig. 2A, DEC1 siRNA considerably reduced the mRNA level of DEC1, whereas, scrambled siRNA had no effect on the expression of DEC1. Notably, ablation of endogenous DEC1 by siRNA resulted in enhanced claudin-1 expression at the mRNA level in both MCF-7 and MDA-MB-231 cells. Consistently, the protein expression of claudin-1 was also upregulated upon DEC1 knockdown in both cell lines (Fig. 2B). Taken together, 


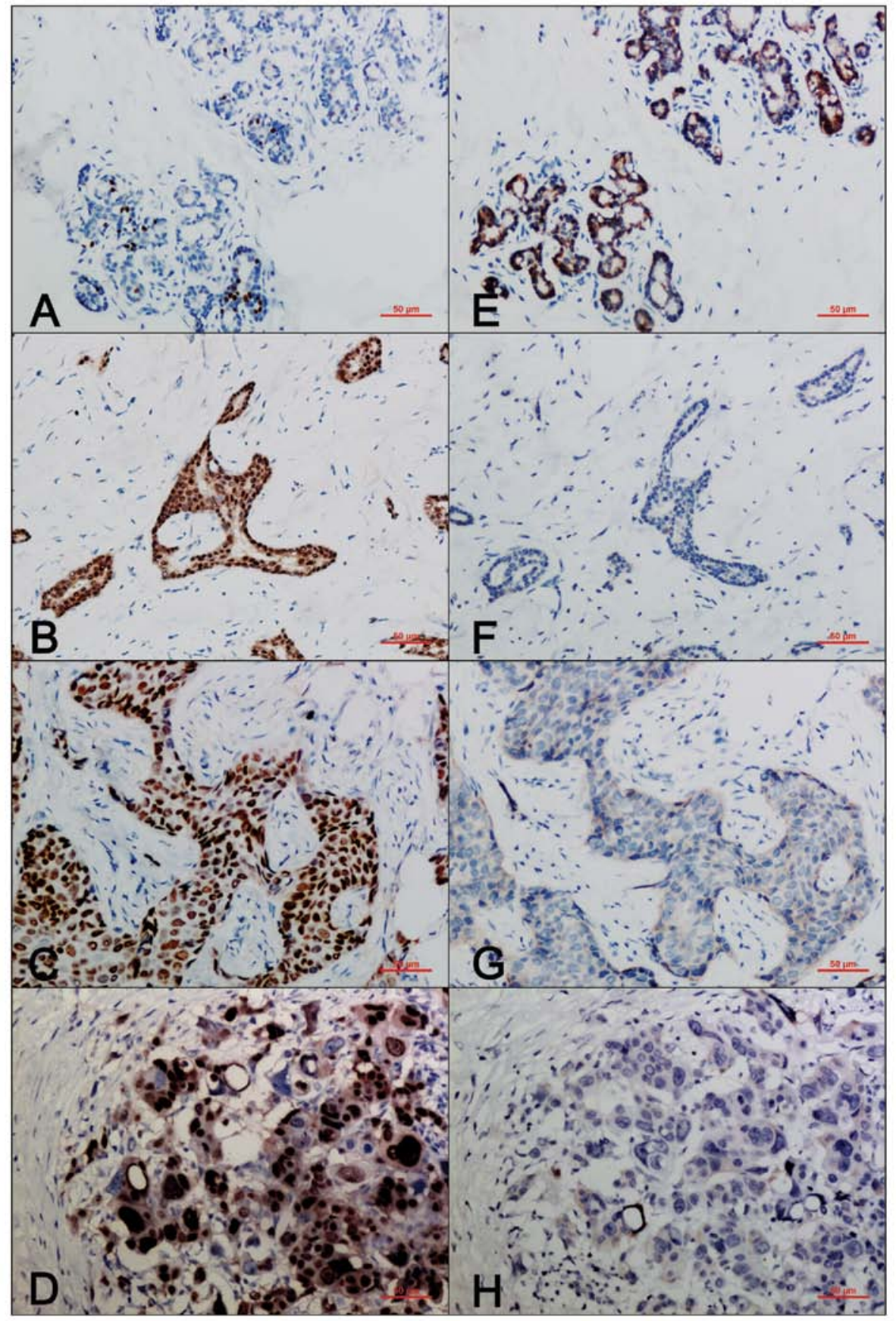

Figure 1. DEC1 and claudin-1 expression in normal breast tissue and invasive ductal carcinoma. (A) The expression of DEC1 in normal breast tissue is patchy and weak, predominantly in luminal epithelial cell nucleoli of normal acini. In contrast, strong nuclear and weak cytoplasmic staining of DEC1 is shown in invasive ductal carcinoma of different grades [(B) grade 1, (C) grade 2 and (D) grade 3]. (E) The expression of claudin-1 in normal breast tissue is noted in the cytoplasm and on the membrane of luminal epithelial cells of normal acini. In contrast, weak or absent cytoplasmic staining of claudin-1 is observed in invasive ductal carcinoma of different grades [(F) grade 1, (G) grade 2 and $(\mathrm{H})$ grade 3]. Scale bars, $50 \mu \mathrm{m}$. Images were captured at a magnification of $\mathrm{x} 400$.

these data imply that DEC1 downregulates claudin-1 expression in breast cancer cell lines. These data confirm that DEC1 downregulates claudin-1 expression and thereby results in the progressive invasiveness of breast cancer.

Role of DEC1 in the invasiveness of breast cancer cells. Breakdown of cell-cell interactions and downregulation of junctional proteins are key steps in tumor invasion. Therefore, we employed the Matrigel invasion assay to test whether the expression level of DEC1 had any impact on cell invasive capability. In both MCF-7 and MDA-MB-231 cells, downregulation of DEC1 by siRNA knockdown resulted in a dramatic reduction in the cell invasive capability compared to the control group (Fig. 3). These data further support the postulate that $\mathrm{DEC1}$ is closely linked to the invasive capability of breast cancer. Together with the observations that DEC1 and claudin-1 are inversely correlated in invasive ductal carcinomas and that DEC1 modulates the claudin-1 expression level, our data suggest that DEC1 may promote breast cancer invasion through its regulation of claudin-1. 
A

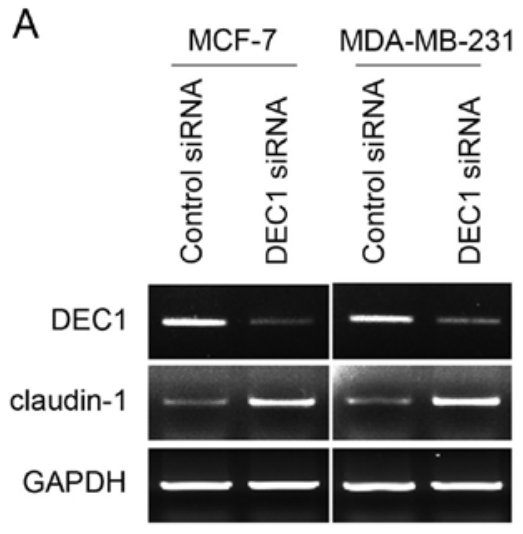

B

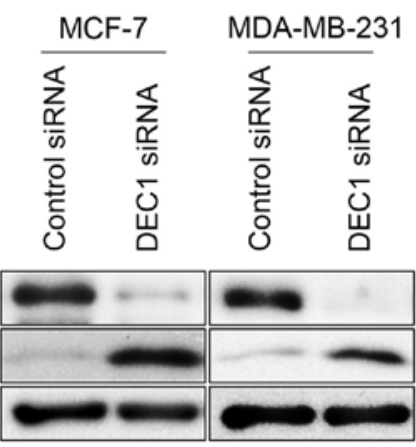

Figure 2. DEC1 knockdown increases claudin-1 expression in MCF-7 and MDA-MB-231 cells. MCF-7 and MDA-MB-231 cells were transfected with control siRNA or siRNA against DEC1 for $48 \mathrm{~h}$. (A) The mRNA levels of claudin-1 were upregulated by DEC1 knockdown in both MCF-7 and MDA-MB-231 cells. (B) The protein expression of claudin-1 was elevated upon DEC1 knockdown in both MCF-7 and MDA-MB-231 cells. Each experiment was performed in triplicate.
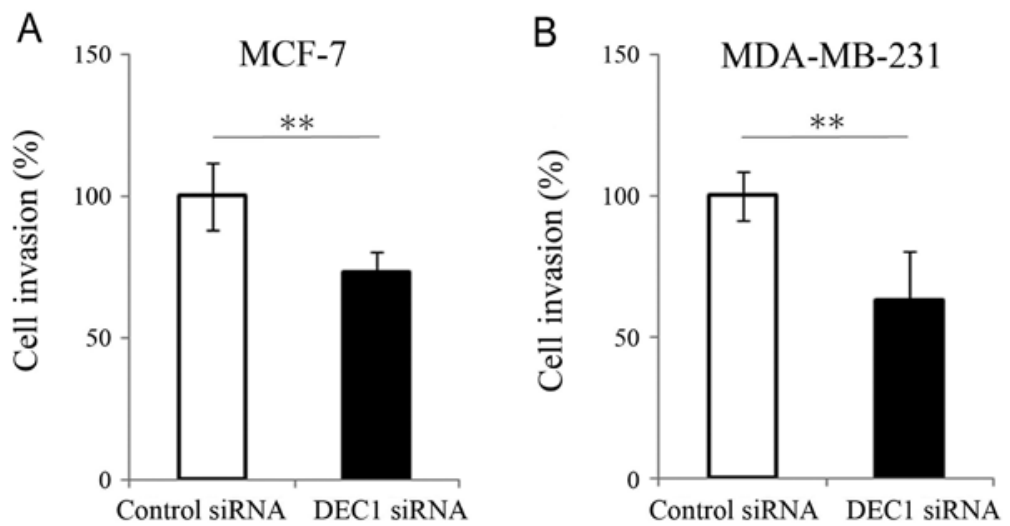

Figure 3. Invasiveness of MCF-7 and MDA-MB-231 cells was analyzed by Matrigel invasion assay. DEC1 siRNA reduced the invasiveness in both (A) MCF-7 and (B) MDA-MB-231 cells ( $\left({ }^{* *} \mathrm{P}<0.01\right)$.

\section{Discussion}

DEC1 is involved in the control of proliferation, differentiation and apoptosis of various types of cells (2). Overexpression of DEC1 was reportedly correlated with a wide array of cancers $(7,12,19,21,22)$. Chakrabarti et al (12) found that the expression of DEC1 increased upon progression from normal tissues to invasive breast carcinomas. Agreeably, in this study, we also observed increased DEC1 expression in breast invasive ductal adenocarcinoma. In addition, the results of our Matrigel invasion assay revealed that DEC1 may be involved in breast cancer invasion. Since the breakdown of cell-cell interaction and downregulated expression of junctional proteins are key steps in tumor invasion, it is possible that the upregulation of DEC1 may have an inhibitory effect on the expression of junctional proteins.

Tight junctions are the most apical of intercellular junctions and appear as a network of continuous filaments on the protoplasmic face of the plasma membrane (23). They contribute to the transepithelial barrier that controls the transport of ions and small molecules through the paracellular pathway $(24,25)$. Claudins are the major component of the tight junction, and to date, 24 claudin family members have been identified (26). Loss of claudin-1, a member of the tight junction, is associated with cancer invasion and the acquisition of the metastatic phenotype $(13,14)$. Functional studies suggest that claudin-1 may be a key player in the progression of breast cancer (18). According to our results, loss of claudin-1 was correlated with lymph nodal metastasis and ER-positive status. Regarding the correlation between ER status and claudin-1, our result is consistent with the findings reported by Blanchard et al (27). Their study also showed that, in ER-negative invasive breast cancers, a higher number of cases were claudin-4 positive, whereas fewer cases were claudin-3 positive. Taken together, this suggests that different claudins have unique expression patterns in different subtypes of breast cancers.

Our clinical data provide the evidence that there is a strong negative correlation between DEC1 and claudin-1 expression in invasive ductal breast carcinomas. We also found that knockdown of endogenous DEC1 enhanced the expression of claudin-1 at both the mRNA and protein levels. DEC1, a transcriptional factor, has been shown to regulate the transcription of target genes by binding to the E-box elements $(28,29)$. There are two E-box motifs in the promoter of claudin-1 (30). Thus, it is likely that DEC1 may bind to the E-boxes in the promoter of claudin-1 and regulate its transcription. Further investigation by us will be directed towards further addressing the mechanism. 
Kon et al (31) along with our previous study demonstrated that DEC1, as a downstream target gene, was induced by transforming growth factor- $\beta$ (TGF- $\beta$ ) and tumor necrosis factor- $\alpha$ (TNF- $\alpha$ ) (32). TNF- $\alpha$ and TGF- $\beta$ were widely reported as regulators in epithelial-mesenchymal transition (EMT) through activation of $N F-\kappa B$ and TGF- $\beta$ signal pathway, respectively $(11,33)$. Notably, TNF- $\alpha$ can also induce the expression of claudin-1 (34), while TGF- $\beta$ increases tumor-initiating cells in 'claudin-low' breast cancer cell lines (35). Based on these results, it is possible that the regulation of claudin-1 by DEC1 may be a bridge which links TNF- $\alpha /$ TGF- $\beta$ and the induction of EMT.

In conclusion, both DEC1 and claudin-1 are closely related with tumorigenesis of breast cancer. DEC1 is positively associated with tumor grade in invasive breast cancer and is negatively correlated with the expression of claudin- 1 . DEC1 may influence the progression of invasive breast cancer through its regulation of claudin-1.

\section{Acknowledgements}

We are grateful to Dr Xiaoman Li for the help in preparing the manuscript.

\section{References}

1. Ivanova A, Liao SY, Lerman MI, Ivanov S and Stanbridge EJ: STRA13 expression and subcellular localisation in normal and tumour tissues: implications for use as a diagnostic and differentiation marker. J Med Genet 42: 565-576, 2005.

2. Shen M, Kawamoto T, Yan W, et al: Molecular characterization of the novel basic helix-loop-helix protein DEC1 expressed in differentiated human embryo chondrocytes. Biochem Biophys Res Commun 236: 294-298, 1997.

3. Nakashima A, Kawamoto T, Honda KK, et al: DEC1 modulates the circadian phase of clock gene expression. Mol Cell Biol 28: 4080-4092, 2008

4. Sato F, Bhawal UK, Kawamoto T, et al: Basic-helix-loop-helix (bHLH) transcription factor DEC2 negatively regulates vascular endothelial growth factor expression. Genes Cells 13: 131-144, 2008.

5. Liu Y, Sato F, Kawamoto T, et al: Anti-apoptotic effect of the basic helix-loop-helix (bHLH) transcription factor DEC2 in human breast cancer cells. Genes Cells 15: 315-325, 2010

6. Bhawal UK, Sato F, Arakawa Y, et al: Basic helix-loop-helix transcription factor DEC1 negatively regulates cyclin D1. J Pathol 224: 420-429, 2011.

7. Li Y, Zhang H, Xie M, et al: Abundant expression of Dec1/ stra13/sharp2 in colon carcinoma: its antagonizing role in serum deprivation-induced apoptosis and selective inhibition of procaspase activation. Biochem J 367: 413-422, 2002.

8. Boudjelal M, Taneja R, Matsubara S, Bouillet P, Dolle P and Chambon P: Overexpression of Stra13, a novel retinoic acidinducible gene of the basic helix-loop-helix family, inhibits mesodermal and promotes neuronal differentiation of P19 cells. Genes Dev 11: 2052-2065, 1997.

9. Turley H, Wykoff CC, Troup S, Watson PH, Gatter KC and Harris AL: The hypoxia-regulated transcription factor DEC1 (Stra13, SHARP-2) and its expression in human tissues and tumours. J Pathol 203: 808-813, 2004.

10. Shi XH, Zheng Y, Sun Q, et al: DEC1 nuclear expression: a marker of differentiation grade in hepatocellular carcinoma. World J Gastroenterol 17: 2037-2043, 2011

11. Xu Q, Ma P, Hu C, et al: Overexpression of the DEC1 protein induces senescence in vitro and is related to better survival in esophageal squamous cell carcinoma. PLoS One 7: e41862, 2012.

12. Chakrabarti J, Turley H, Campo L, et al: The transcription factor DEC1 (stra13, SHARP2) is associated with the hypoxic response and high tumour grade in human breast cancers. Br J Cancer 91: 954-958, 2004
13. Tokés AM, Kulka J, Paku S, et al: Claudin-1, -3 and -4 proteins and mRNA expression in benign and malignant breast lesions: a research study. Breast Cancer Res 7: R296-R305, 2005.

14. Swisshelm K, Macek R and Kubbies M: Role of claudins in tumorigenesis. Adv Drug Deliv Rev 57: 919-928, 2005.

15. Sheehan GM, Kallakury BV, Sheehan CE, Fisher HA, Kaufman RP Jr and Ross JS: Loss of claudins-1 and -7 and expression of claudins-3 and -4 correlate with prognostic variables in prostatic adenocarcinomas. Hum Pathol 38: 564-569, 2007.

16. Cohn ML, Goncharuk VN, Diwan AH, Zhang PS, Shen SS and Prieto VG: Loss of claudin-1 expression in tumor-associated vessels correlates with acquisition of metastatic phenotype in melanocytic neoplasms. J Cutan Pathol 32: 533-536, 2005.

17. Chao YC, Pan SH, Yang SC, et al: Claudin-1 is a metastasis suppressor and correlates with clinical outcome in lung adenocarcinoma. Am J Respir Crit Care Med 179: 123-133, 2009.

18. Myal Y, Leygue E and Blanchard AA: Claudin 1 in breast tumorigenesis: revelation of a possible novel 'claudin high' subset of breast cancers. J Biomed Biotechnol 2010: 956897, 2010.

19. Giatromanolaki A, Koukourakis MI, Sivridis E, et al: DEC1 (STRA13) protein expression relates to hypoxia-inducible factor 1-alpha and carbonic anhydrase-9 overexpression in non-small cell lung cancer. J Pathol 200: 222-228, 2003.

20. Morohashi S, Kusumi T, Sato F, et al: Decreased expression of claudin-1 correlates with recurrence status in breast cancer. Int J Mol Med 20: 139-143, 2007.

21. Ivanova AV, Ivanov SV, Danilkovitch-Miagkova A and Lerman MI: Regulation of STRA13 by the von Hippel-Lindau tumor suppressor protein, hypoxia, and the UBC9/ubiquitin proteasome degradation pathway. J Biol Chem 276: 15306-15315, 2001.

22. Li Y, Xie M, Yang D, et al: The expression of antiapoptotic protein survivin is transcriptionally upregulated by DEC1 primarily through multiple sp1 binding sites in the proximal promoter. Oncogene 25: 3296-3306, 2006.

23. Anderson JM and Van Itallie CM: Physiology and function of the tight junction. Cold Spring Harb Perspect Biol 1: a002584, 2009.

24. Diamond JM: Twenty-first Bowditch lecture. The epithelial junction: bridge, gate, and fence. Physiologist 20: 10-18, 1977.

25. Tobioka H, Isomura H, Kokai Y, Tokunaga Y, Yamaguchi J and Sawada N: Occludin expression decreases with the progression of human endometrial carcinoma. Hum Pathol 35: 159-164, 2004.

26. Tsukita S, Furuse $M$ and Itoh M: Multifunctional strands in tight junctions. Nat Rev Mol Cell Biol 2: 285-293, 2001.

27. Blanchard AA, Watson PH, Shiu RP, et al: Differential expression of claudin 1,3 , and 4 during normal mammary gland development in the mouse. DNA Cell Biol 25: 79-86, 2006.

28. Li Y, Xie M, Song X, et al: DEC1 negatively regulates the expression of DEC2 through binding to the E-box in the proximal promoter. J Biol Chem 278: 16899-16907, 2003.

29. St-Pierre B, Flock G, Zacksenhaus E and Egan SE: Stra13 homodimers repress transcription through class B E-box elements. J Biol Chem 277: 46544-46551, 2002.

30. Martinez-Estrada OM, Cullerés A, Soriano FX, et al: The transcription factors Slug and Snail act as repressors of Claudin-1 expression in epithelial cells. Biochem J 394: 449-457, 2006.

31. Kon N, Hirota T, Kawamoto T, Kato Y, Tsubota T and Fukada Y: Activation of TGF-beta/activin signalling resets the circadian clock through rapid induction of Decl transcripts. Nat Cell Biol 10: 1463-1469, 2008.

32. Liu Y, Wang L, Lin XY, et al: Anti-apoptotic effect of claudin-1 on TNF- $\alpha$-induced apoptosis in human breast cancer MCF-7 cells. Tumour Biol 33: 2307-2315, 2012.

33. Wu Y, Sato F, Yamada T, et al: The BHLH transcription factor DEC1 plays an important role in the epithelial-mesenchymal transition of pancreatic cancer. Int J Oncol 41: 1337-1346, 2012.

34. Kondo J, Sato F, Kusumi T, et al: Claudin-1 expression is induced by tumor necrosis factor- $\alpha$ in human pancreatic cancer cells. Int J Mol Med 22: 645-649, 2008.

35. Bruna A, Greenwood W, Le Quesne J, et al: TGF $\beta$ induces the formation of tumour-initiating cells in claudin ${ }^{\text {low }}$ breast cancer. Nat Commun 3: 1055, 2012. 